De Boeck, S., Van Hertem, D. (2015). Under Frequency Load Shedding Schemes in Systems with High PV Penetration: Impact and Improvements. Proc. IEEE Powertech 2015, Eindhoven, Netherlands, 29 June -2 July 2015, 6 pages.

Digital Object Identifier:

$\underline{10.1109 / P T C .2015 .7232605}$

URL:

http://ieeexplore.ieee.org/stamp/stamp.jsp?tp=\&arnumber=7232605

(C2015 IEEE. Personal use of this material is permitted. Permission from IEEE must be obtained for all other users, including reprinting/ republishing this material for advertising or promotional purposes, creating new collective works for resale or redistribution to servers or lists, or reuse of any copyrighted components of this work in other works. 


\section{Under Frequency Load Shedding Schemes in Systems with High PV Penetration: Impact and Improvements}

\author{
Steven De Boeck \\ Graduate Student Member, IEEE \\ Department of Electrical Engineering, ELECTA \\ KU Leuven, Belgium \\ steven.deboeck@esat.kuleuven.be
}

\author{
Dirk Van Hertem \\ Senior Member, IEEE \\ Department of Electrical Engineering, ELECTA \\ KU Leuven, Belgium \\ Dirk.VanHertem@esat.kuleuven.be
}

\begin{abstract}
With the increasing integration of renewable energies in the power system a thorough analysis of the adequacy of defence plans is needed. This paper focusses on the impact of a high penetration of photovoltaics in distribution systems on the under frequency load shedding schemes designed by the TSO. First an overview of the issues arising from a higher penetration of embedded renewable energy sources in the current UFLS scheme design is described. It will be shown that neglecting photovoltaics in load shedding scheme design can lead to adverse effects in system stability and can have a disproportional high social impact. Subsequently an overview of different methodologies to improve the UFLS scheme design is given. These methodlogies are then applied to the measurements of two feeders in the Belgian power system. From the evaluation of these results, it can be concluded that the proposed methodologies lead to substantial improvements in UFLS scheme adequacy.
\end{abstract}

Index Terms-Under Frequency Load Shedding Schemes, Distributed generation, Photovoltaics, Defence Plan

\section{INTRODUCTION}

The EU is targeting RES to provide up to $34 \%$ of electricity production by 2020 and is targetting a reduction of the greenhouse gas emissions by $80-95 \%$ by 2050 . [1]-[3] To stay on track in realizing this greenhouse gas reduction, the share of RES should further increase to $50-60 \%$ by 2030. [4] Although RES contributes significantly to the climate goals, they also cause a paradigm shift in system operations. System operation is becoming increasingly variable and uncertain. One aspect is the impact and role of the increasing penetration of distributed generation (DG) on the European Defence Plans. These DG units are characterized by their fluctuating power output, and in general provide no inertia to the system. This is because they are usually connected to the grid using a power electronics converter. Moreover, these units are mostly installed in the distribution grid, whereas the defence plans and load shedding schemes are coordinated on a transmission system operator (TSO) level. Two problems arise from this. Firstly, the TSO has only a limited view on the actual state of the power system as the DSO load which is seen from the TSO side hides the internal generation. Secondly, the system behaves differently

The research leading to these results has received funding from the European Union Seventh Framework Programme under grant agreement nº283012 than it did before due to a considerable decrease in system inertia. As a consequence, the under frequency load shedding (UFLS) scheme, in which predefined feeders are disconnected from the grid, needs to be adapted. [5] The reason is that not only load, but also the distributed generation will be disconnected. [6] The total lost load is the sum of the load seen by the transmission system operator and the load masked by local generation. The resulting disconnected load might therefore be considerably higher than expected, leading to a disproportional high social impact (ENS). Also, the traditional selection of curtailment is based on historic data and thus does not take the actual system state into account [7]. Nevertheless, the actual, momentary consumption might differ considerably from the expected one (e.g. due to solar generation), and the under frequency load shedding equipment might not be as effective as planned. This can lead to frequency deviations which exceed the expected ones and therefore can even result in further load shedding or even a black-out. Hence, it is important for the TSO to have a clear overview at any time of the amount of power produced by the DG units at the different feeders and to include this information in the load shedding scheme/defence plan. Such a scheme might be adaptive, to take into account the seasonal and daily variations of the DG to load ratio.

\section{Typical Mixed Feeder LoAd Profile}

Due to the integration of more photovoltaic generation in the distribution system, the typical load profile measured at the beginning of the feeder has changed significantly. In many residential areas photovoltaic panels have been installed on rooftops. The typical generation curve for photovoltaics is given in Fig.1 (dotted line). During the night the photovoltaic generation is zero. Between sunrise and sunset the generation curve can be approximated by a half sine curve with the maximum around noon (not taking clouds into account). The load measured (dashed line) by the distribution system operator at the beginning of the feeder, is the summation of the PV generation curve and the real load curve(full line). This measured load curve is given in Fig.1. It can be seen that part of the actual load is being masked by the distributed generation installed on the feeder. Depending on the amount of installed 
photovoltaic panels and the amount of solar irradiation, the measured load curve can become negative during certain hours in the day Fig.1 (between 11:00 and 14:30). This means that the local generation on the feeder is higher than the load. If the UFLS scheme would be activated during these hours, generation will be disconnected instead of load. Even if the measured load curve is not negative, the total impact of disconnecting this feeder is much larger. First of all, the real load disconnected can be many times higher than the measured load. Secondly the customer is unable to inject power to the system from his photovoltaic panels. This causes the customer to lose money, as in many European countries subsidies are given for every MWh injected into the system.

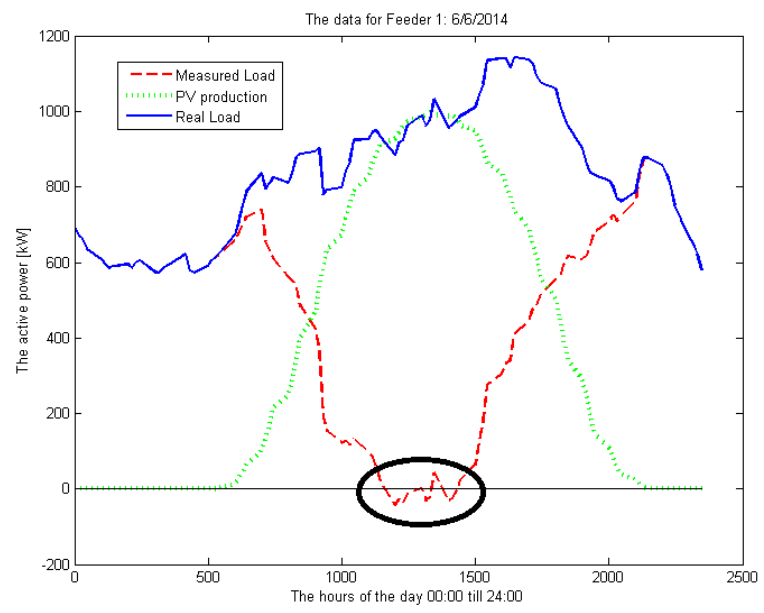

Fig. 1. Measurements on a mixed feeder (6/6/2014)

\section{Current Under Frequency LoAd Shedding SCHEME DESIGN}

Traditionally the design of the UFLS scheme and the determination of the set points for the under frequency relays takes place long before the UFLS scheme is being activated. The set points are usually based on the historical data, as a representative day is chosen for each of the seasons. One of the difficulties in designing this scheme remains the fact, that it is difficult to know the exact amount of load that will be connected at the time of the activation of the UFLS scheme. As shown in Fig. 1 with the integration of more and more photovoltaics in the distribution system, this is getting even more difficult. The pattern now changes both with the load behaviour as with the renewable generation behaviour.

Generally when designing an UFLS scheme, the following aspects are taken into account:

- The type of scheme: is chosen to re-establish the power blance by disconnecting a suitable amount of load for considered contingencies, and thereby determines the number of load shedding steps and their activation frequency [8]. Overall, the distinction between linear, early and late schemes can be made [9]. The scheme ENTSOE advices to the transmission system operator is a linear scheme [10].

- Priority ranking of feeders: The feeders are ranked based on the type of load connected. Generally rural residential feeders are the first to be disconnected, while feeders connecting hospitals, communication infrastructure, etc. are normally last.

- Equal geographic spreading: The equal geographic spreading is pursued to avoid large changes in power flow between different areas of the power system at the activation of the UFLS scheme. This can be to minimize the change in power flow within one TSO area as well as between two TSO areas.

- Distributed generation: If the distributed generation is connected via a designated feeder, than the priority of this feeder is considered to be higher than load feeders. Therefore the feeder will not be disconnected. Though the majority of the feeders are mixed feeders, with both local generation and load. The renewable generation on those feeders is currently not taken into account during the UFLS scheme design. With increasing amount of PV panels installed, this is becoming increasingly important.

\section{Test Case Feeders}

The different methodologies, which will be presented in the next section have been applied to two distribution feeders in the Belgian power system. The measurements of the data of these feeders took place from the beginning of April 2014 till the end of August 2014. An overview of the general data and composition of the two feeders is given in Table I. The data used for the PV production in the different figures, is based on the PV measurements of the province were the feeders are located.

TABLE I

General FEEDER DATA

\begin{tabular}{lrr}
\hline & Feeder 1 & Feeder 2 \\
\hline Type of load & Residential & Residential \\
Number of cabins & 10 & 5 \\
Installed PV(kW) & 1264 & 188 \\
Start Measurements & $1 / 04 / 2014$ & $1 / 04 / 2014$ \\
End Measurements & $31 / 08 / 2014$ & $31 / 08 / 2014$ \\
Peak Load(kW) & 1425 & 310 \\
Minimal Loading(kW) & -43.44 & 18.50 \\
\hline
\end{tabular}

\section{IMPACt of Different Under Frequency LoAD SHEDDING SCHEME}

Several methodologies will be proposed to improve the UFLS scheme design. To compare different UFLS methodologies, it is important to define a good metric. An overview of different metrics is given, from which the most important ones will be selected. Hereafter several methodologies are defined to determine the ranking of the feeders in the UFLS. 


\section{A. Load Dependened Settings}

In this approach, the priority of feeders is determined by the type of load connected to each of the feeders. This is the current practice. Low priority feeders (e.g. rural areas) will be disconnected before higher priority feeders (e.g. densely populated areas). As both feeders described in Table 1 have only residential load connected, the priority of both feeders is equal. As mentioned earlier, once a type of UFLS scheme has been chosen, the feeders in the system have to be divided over the different steps in the scheme. Each step should disconnect a predefined amount of load. E.g. ENTSOE advices to disconnect $10 \%$ of the load in the TSO control area in the first step of the scheme, when the frequency drops below $49 \mathrm{~Hz}$. Up till now, the number of feeders that should be placed behind this relay to achieve this $10 \%$ of load, is based on the mean value and standard deviation of the historical load data of this feeder. From this data mean values of load can be calculated over a specific period of time, but often a specific day in each of the seasons is used to make this estimation. With the increased integration of distributed generation, the feeder characteristic is changing daily.

In Fig.2 the load distribution of feeder one with (right) and without (left) PV is given for the months April till the end of August. The first important difference between the two distributions, when taking the PV into account, is that the measured load can become negative. This means that the feeder is injecting power to the transmission system. Secondly the peak for load values of around $600 \mathrm{~kW}$ is almost the same in both figures. These data points mainly represent consumption during the night, when there is no PV generation. Thirdly it can be seen that for the measured load, the number of data points between $1000 \mathrm{~kW}$ and $1300 \mathrm{~kW}$ is considerably lower than when there would be no PV (left). Normally the consumption during the day on a residential feeder is higher than during the night. Though the power
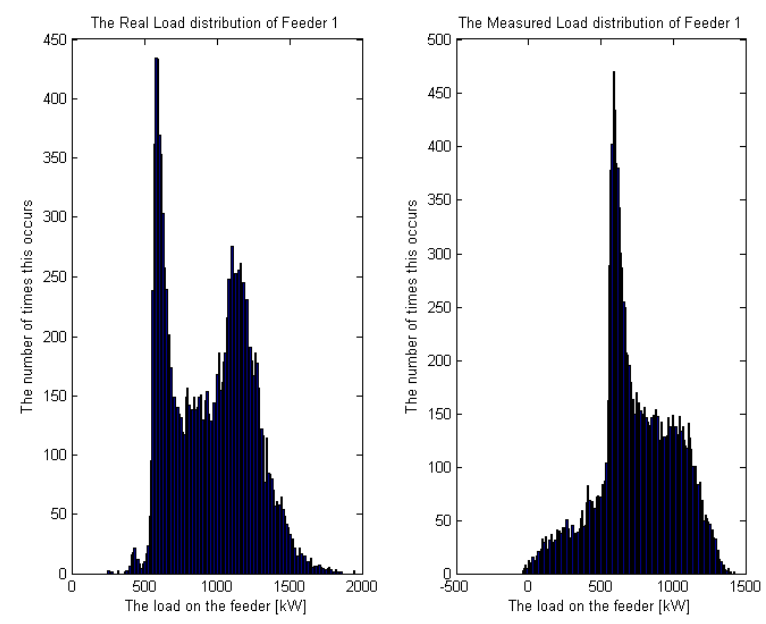

Fig. 2. Load distribution of feeder 1 ( $1^{\text {st }}$ April $2014-31^{\text {st }}$ August 2014): real load(left), measured (load right) generated by the PV masks these loads and shifts the data points towards lower values, which can be clearly seen in 2 (right), as the number of data points with values below $900 \mathrm{~kW}$ have increased. Certainly the number of data points below $500 \mathrm{~kW}$ has significantly increased. So determining the number of feeders that should be placed within each step of the UFLS scheme based only on values like the mean value and standard deviation of the load will give a misleading image of the real load connected to the feeder. This can be seen by comparing Table 2 with Fig.2. The mean value of the measured load (with PV) is significantly smaller (739.3 $\mathrm{kW}$ ), compared to the mean value of real load connected to the feeder $(957.2 \mathrm{~kW})$. The standard deviation is only slightly changed.

TABLE II

MEAN AND STANDARD DEVIATION VALUES FOR FEEDER 1

\begin{tabular}{lrr}
\hline Feeder 1 & Mean & Standard Deviation \\
\hline Real Load (no PV)[kW] & 975.2 & 280.6 \\
Measured Load (with PV)[kW] & 739.3 & 267.6 \\
\hline
\end{tabular}

The overall result of applying this method in the UFLS scheme design will result in more feeders to be placed behind a relay with a higher frequency threshold. Recalling the example of disconnecting $10 \%$ of load at $49 \mathrm{~Hz}$ : more feeders will be disconnected at $49 \mathrm{~Hz}$ to make sure that the $10 \%$ of load will be reached. But with this methodology it is still possible to disconnect feeders with more generation than load (e.g. sunny day), which would worsen the system stability. But the contrary is also possible, namely on a cloudy day or during the night, more load than expected might be disconnected, resulting in overcompensation of the under frequency event.

\section{B. Direction of Current}

As described before, the TSO's main concern is to avoid the disconnection of generation as this would aggravate the under frequency condition by further decreasing the system frequency. Therefore an extra check can be integrated in the UFLS relay, namely the direction of the current flowing through the relay. The main advantage of this method is that it makes use of the measurement of local parameters, namely frequency and current. If a specific feeder has more generation than load connected, the current will flow towards the medium voltage (MV) system. In Fig.1 it can be seen that for feeder 1 this situation could take place between 11:30 and 14:30 hours on the 6th of June 2014 (the measured load becomes negative). If there is more load than generation connected, the current will flow from the MV system to the low voltage feeder connecting the customers. This is in Fig.1 the case before 11:30 and after 14:30. During these hours the actual load can still be bigger than the measured load. This is not taken into account in this method and is therefore a drawback.

Implementing this methodology requires from the TSO to replace the currently installed UFLS relays on feeders with significant amounts of distributed generation installed. 


\section{Periodic Settings (Seasonal \Day and Night)}

With a focus on the integration of photovoltaics in the distribution system, a logical methodology is to change the settings of the UFLS scheme depending on the expected irridiation. During the day feeders with more photovoltaics will have a higher priority than feeders with only load. At night this priority ordering can be inversed. This methodology will thus change the priority of the residential feeders in the first steps of the load shedding scheme depending on whether it is day or night. As described in previous sections, during the day the PV generation will mask part of the load connected to the feeder, resulting in a reduced load measured. Therefore the feeders need to be ranked to minimize the impact, while attaining the contribution needed to stabilize the under frequency event taking place in the transmission system. A possible method to rank the feeders is based on the index presented in (1):

$$
\text { Index }=\frac{\text { Installed PV Power }}{\text { Historical Measured Load }}
$$

This equation gives the ratio of the installed photovoltaic power over the historic measured load. The installed photovoltaic power is generally known by the different DSOs, as the consumer needs to declare this installed power to be able to receive subsidies and approval to connect the installation to the system. The TSO should negotiate with the DSO on exchanging this data to be able to perform the presented analysis. For the denominator several options are possible. One option is to use the peak power measured on the feeder the year before during the day time (6:00-21:00). In M1 (2), the index is equal to the installed PV on the feeder divided by the peak power measured on the feeder in the same season the year before. This results in a fixed ranking for each season. Another option is to divide the installed PV by a mean value over a specific period of time. This time period should than be taken in accordance with the number of times a year the TSO would like to adjust the settings of the UFLS relay. This can therefore be seasonal, monthly,... . In M2 (3), an average for 1 Month has been chosen.

$$
\begin{gathered}
M 1=\frac{\text { Installed PV Power }}{\text { Measured Load } \text { Peak Power }(\text { season })} \\
M 2=\frac{\text { Installed PV Power }}{\text { Measured Load }} \text { Average Power }(1 \text { month })
\end{gathered}
$$

These indices can then be used to define a disconnection ranking for the different feeders. For both index M1 and M2, the feeder with the smallest index value will be disconnected first, as the impact of the load masking by the PV on this feeder will be smallest. The results for applying these indices during the day time on the feeders 1 and 2 (earlier described) are presented in Fig.3. The X-axis numbers represent the quarter hour time intervals during the day time for the months April till August. The index M1 is a constant value for both feeders 1 and 2, as the installed PV is considered not to be changed and the denominator of M1 is constant (measured peak load). Therefore according to M1 the ranking of the feeders is constant over the considered time period. The respective M1 values for the feeders 1 and 2 are 0.9 and 0.6. The feeder 2 has a smaller M1 value and will therefore be disconnected earlier than feeder 1. From Fig.3, it is clear that the index M2 changes for each quarter hour time interval for both feeders. Though the feeder ranking is also fixed here, as the index M2 for feeder 2 is always smaller than for feeder 1 . But it is important to notice that the spread between the two feeders is not constant. Therefore the ranking of feeders can change.

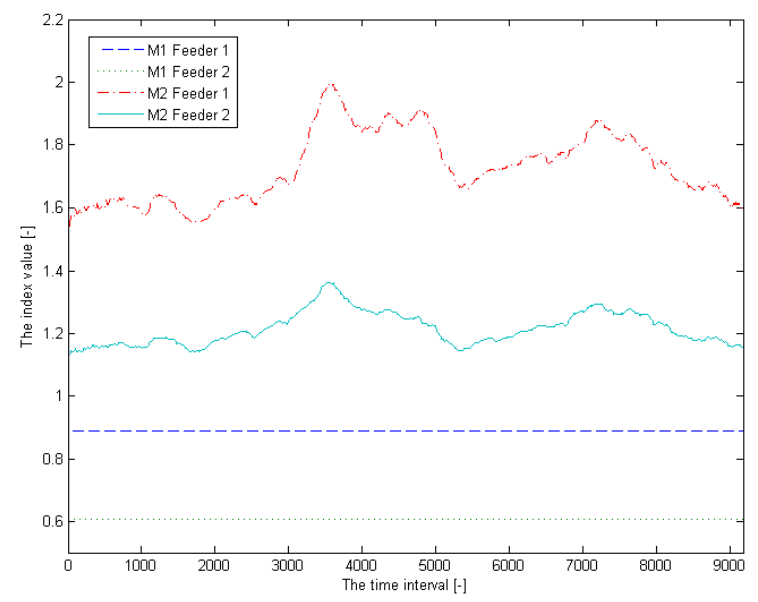

Fig. 3. Feeder ranking using the indices M1 and M2

The use of historical data is interesting in the current framework, as the relays cannot be changed remotely. Therefore this approach provides a possibility to optimize the feeder ranking season by season. Instead of using the historical load data, it is also possible to incorporate an estimated load profile for the next day or month. But communication is then needed to automate the proces, but the expected gain for the system operator is limited.

During the night there is no specific priority among the different residential feeders, as there is no influence of the photovoltaic generation on the feeder behaviour. Therefore a logical approach is to use the inverse priority of feeder disconnection that is used during the day to achieve equal spreading of the burden.

\section{Smart Grid Approach (Intra Day)}

In this method the power off take at the beginning of the feeder is read out close to real time. Based on the PV estimation for the area where the feeders are located, and the exact amount of photovoltaic panels installed, an estimation for the PV production can be made. The index proposed in (4), combines this information to define the efficiency of the load shedding action. This index is defined as the ratio of the contribution to the system stabilization, over the impact of the load shedding action. The contribution to the system stabilization is the amount of load that the TSO sees and 
wants to disconnect to restore the balance between generation and load. Therefore the system contribution is equal to the measured load at the beginning of the feeder. The impact of the load shedding action is equal to the sum of the real load disconnected and the PV generation that is not able to inject into the system. As described earlier, the real load is masked by the PV generation. Therefore the real load connected can be described by the sum of the measured load and the estimation of the PV generation (7).

$$
\begin{gathered}
M 3=\frac{\text { System Contribution }}{\text { Impact }} \text { L action } \\
\text { System Contribution }=\text { Measured Load } \\
\text { Impact }_{L S \text { action }}=\text { Real Load }+P V \text { Generation } \\
\text { Real Load }=\text { Measured Load }+P V \text { Generation }
\end{gathered}
$$

Combining the equations 4 to 7 gives:

$$
M 3=\frac{\text { Measured Load }}{\text { Measured Load }+2 * P V \text { Generation }}
$$

This index can also be applied to the earlier described feeders 1\&2. In Fig.4 the boxplot of the day hour values of M3 for the feeders $1 \& 2$ are given. At night the value of M3 is always one. For feeder 1 it can be seen that there are also negative values (feeder injecting into the system). The mean value of M3 for feeder 2 is higher than the mean value for feeder 1 . Also the quartiles of feeder 2 are higher than of feeder 1. A higher value of M3 means a higher priority for feeder disconnection. In general disconnecting feeder 2 before feeder 1 is preferred. This is the same conclusion as obtained from indices M1\&M2 as is summarised in Table III.

However, when looking at the M3 values over the same day, this order might change. From the load measurements on feeder 1 (Fig.5) and feeder 2 (Fig.6) taken on the same day, the M3 values for both feeders in Fig.7 can be calculated. As described earlier, a higher value for M3 means an earlier disconnection. Therefore, from the disconnection efficiency in Fig.7 the feeder ranking (Fig.8) can be obtained.

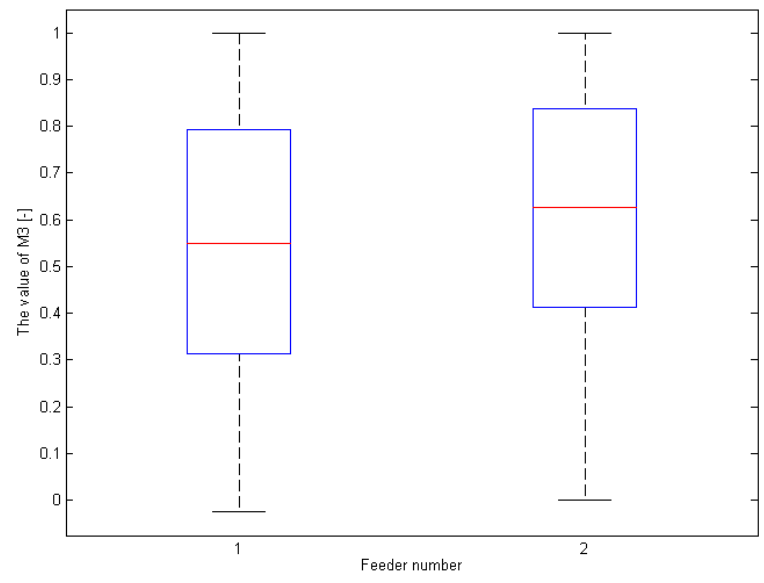

Fig. 4. The distribution of the shedding efficiency for feeders 1 and 2 (day hours)
From these result it is clear that from 6:00 till 11:00 the feeder 1 should be disconnected first, while from 11:00 till 21:00 feeder 2 should be disconnected first to have the maximum contribution to stabilize the system whit minimum impact(ENS). When combining the solar predictions with the cloud predictions in a large area, the proposed feeder ranking can be further optimized, while taking into account local variations in solar production.

TABLE III

FEEDER RANKING BASED ON M1 AND M2

\begin{tabular}{lrr}
\hline $5 / 06 / 2014$ & M1 & M2 \\
\hline Feeder1 & 0.89 & $\sim 1.68$ \\
Feeder2 & 0.61 & $\sim 1.25$ \\
Priority & First Feeder 2 & First Feeder 2 \\
\hline
\end{tabular}

Finally it is important to mention, that to implement index M3, the relays need to be set close to real time (e.g. 15 minute base). Therefore communication with the relays is necessary to be able to set the relays automatically by an algorithm. As most existing relays still need to be set manually, the

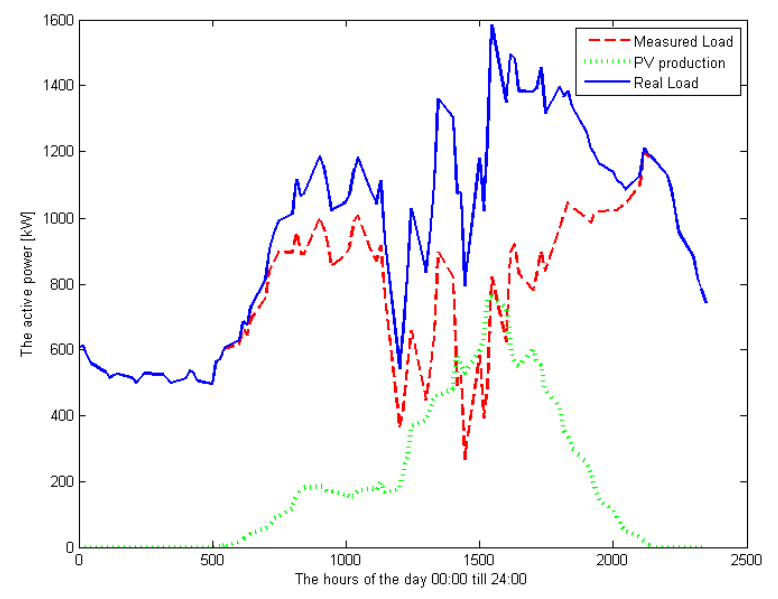

Fig. 5. Measurements for feeder 1

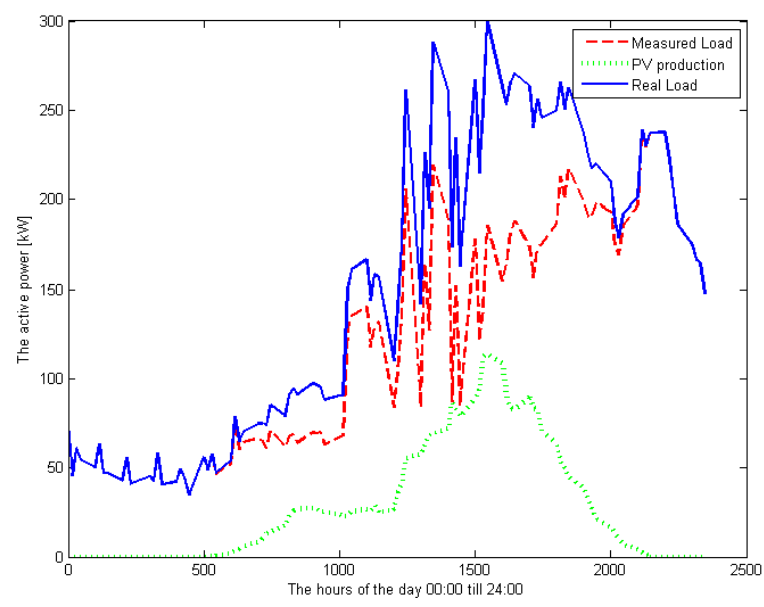

Fig. 6. Measurements for feeder 2 


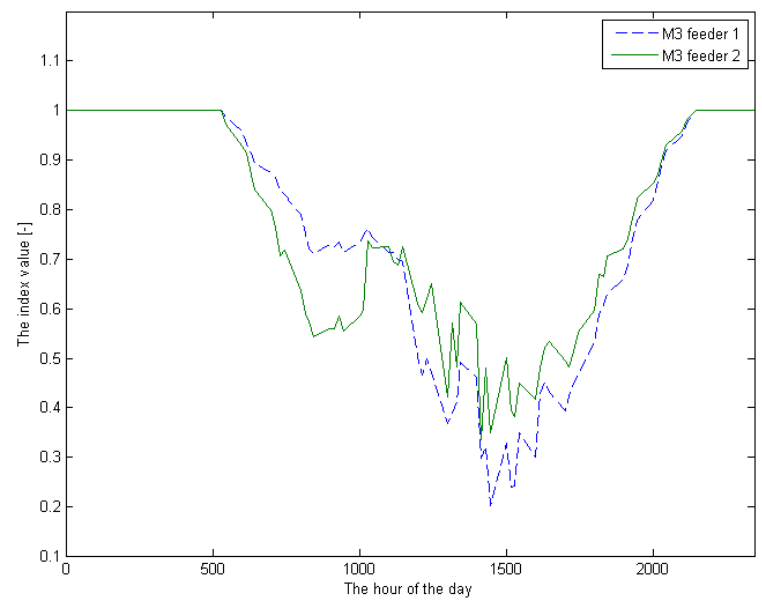

Fig. 7. Disconnection efficiency for feeder 1 and 2

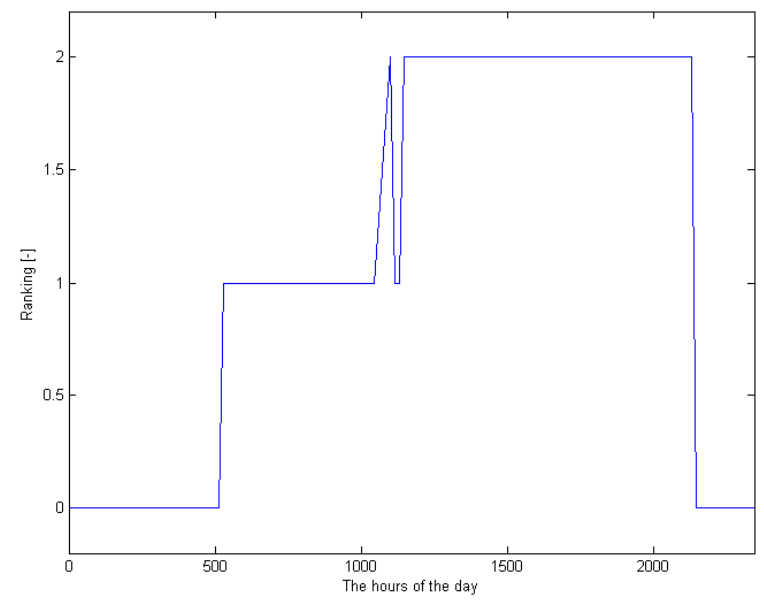

Fig. 8. Ranking of feeders: 0(no priority), 1 (first disconnect feeder 1), 2 (first disconnect feeder 2)

relays should be replaced, to be able to implement this method. This is a costly procedure, though many DSOs and TSOs are already gradually replacing old relays with relays that have these new functionalities.

\section{CONCLUSIONS}

This contribution makes clear that the installed photovoltaics in distribution grids should be taken correctly into account in the design of UFLS schemes. This, firstly to insure that the disconnection of feeders results in the disconnection of an amount of load close to the predefined value in the UFLS. This is of main importance, because if the predefined value is not disconnected by the scheme, more steps of the scheme need to be activated and thus higher priority load will be disconnected. Secondly, when disconnecting feeders with high local generation, both load and generation are disconnected, while only a limited part is actually useful. The current implemented methodology is based on the idea that only load was connected to the feeder, such that disconnection would always lead to a positive contribution in stabilizing the frequency. It is clear that with the changing feeder behaviour, due to embedded generation, the adequacy of the UFLS scheme can no longer be guaranteed under all circumstances. The first possible solution presented in this paper was the directional relay. This relay takes both the frequency and the direction of the current into account. If the current is flowing from the feeder towards the transmission system, then the feeder is not being disconnected as this feeder can be considered as net generation. This methodology would already solve the biggest issue for the TSO, as they want to avoid the disconnection of generation during under frequency events. The second method uses an index based on historical load data and the amount of installed power of photovoltaics. Depending on the number of times a year the TSO wants to adapt the relays, the index can be calculated every season, month,... . But generally changing relay settings remains costly in man-hours. This methodology is extended by inversing the feeder ranking at night to spread the burden equally over the consumers. Finally a smart grid approach has been presented. In this approach the load shedding efficiency of a feeder is calculated close to real time, based on the actual load and an estimation of renewable generation production on that feeder. Thereafter the efficiencies of the different feeder are ranked to provide the relay the exact disconnection sequence. Implementing this methodology implies more intelligent relays, as communication with the relay is necessary. Most of the relays installed today, still need to be set manually. Therefore, these relays would require replacement.

\section{ACKNOWLEDGMENTS}

The authors grathefully acknowledge INFRAX for delivering the data on which this research is based.

\section{REFERENCES}

[1] JRC, "Technical Assessment of the renewable Energy Action Plans," 2011. [Online]. Available: http://ec.europa.eu/dgs/jrc

[2] European Commission, "Energy 2020 A strategy for competitive, sustainable and secure energy," 2010.[Online]. Available: http://eurlex.europa.eu

[3] European Commission,'EU Action Against Climate Change - Leading Global Action to 2020 and Beyond," 2009[Online]. Available: http://www.eskema.eu/

[4] European Climate Foundation (ECF),'Power Perspectives 2030 - On the road to a decarbonised power sector,"2011[Online]. Available: http://www.roadmap2050.eu

[5] Seyedi, H.; Sanaye-Pasand, M., "New centralised adaptive load-shedding algorithms to mitigate power system blackouts," Generation, Transmission \& Distribution, IET , vol.3, no.1, pp.99,114, Jan. 2009

[6] Ying-Yi Hong; Ming-Chun Hsiao; Yung-Ruei Chang; Yih-Der Lee; Hui-Chun Huang, "Multiscenario Underfrequency Load Shedding in a Microgrid Consisting of Intermittent Renewables," Power Delivery, IEEE Transactions on , vol.28, no.3, pp.1610,1617, July 2013

[7] Terzija, V.V. ,'Adaptive underfrequency load shedding based on the magnitude of the disturbance estimation," Power Systems, IEEE Transactions on, vol.21 , no.3 , pp.1260-1266, Aug 2006

[8] Ketabi, A. and Fini, M.H.,"An Underfrequency Load Shedding Scheme for Hybrid and Multiarea Power Systems," Smart Grid, IEEE Transactions on, vol.6, no.1, pp.82-91, Jan 2015

[9] De Boeck S., Van Hertem D., "Considerations and Recommendations for the Harmonisation of Under Frequency Loadshedding Schemes in Multi Zone Meshed Grids," In CIGRE Innovation for secure and efficient transmission grids. Brussels, Belgium, 12-14 March 2014

[10] ENTSO-E, "Operations handbook policy 5: Emergency operations, ', Tech.Rep.,2012.[Online]. Available: https://www.entsoe.eu/publications 